\title{
On the Interaction Between Stimulus Features and Context in the Perception of Causality
}

\author{
Jonathan Fugelsang ${ }^{1, *}$ and Matthew Roser ${ }^{2}$ \\ ${ }^{I}$ Department of Psychology, University of Waterloo, Canada \\ ${ }^{2}$ School of Psychology, University of Plymouth, UK
}

\begin{abstract}
Much research has supported the hypothesis that the perceptual system generates impressions of causality based on simple cues available in the environment. We review evidence that this extraction of causal relations from perceptual cues is likely an automatic property of the visual system akin to other basic perceptual processes, such as perceptual grouping and illusory contour completion. We posit that it is this automatic characteristic of perceptual causality that underlies the proliferation of context effects associated with perceived causal events. Here, the presence of a perceived causal relationship may impact the perception of other features of the causal stimulus and other stimuli surrounding the causal event. We discuss current research, and present future research directions that promise to uncover some of the mechanisms underlying how causality is attributed from, and changes how we perceive and respond to, simple low-level stimuli in the environment. Such research will enrich our understanding of how the perception of causally-relevant stimulus features interacts with their context to enable us to effectively perceive, understand, and act upon our environment.
\end{abstract}

Keywords: Perceptual Causality, Context, Automaticity, Contiguity.

\section{INTRODUCTION}

One of the most fundamental attributes of the human mind is its ability to perceive and interpret causal relations from information in the environment. Indeed, the detection of causal relations is a key ability underlying an individual's success in the dynamic world in which we live. Much research has demonstrated that causal links are often induced from observations of dynamic interactions between simple low-level stimuli. A classic example is the well-known launching effect. Here, if an object moves immediately after perceived contact is made by a preceding object, the motion of the second object is reported by the majority of observers to have been caused by the first. Importantly, this percept is generated even when the stimuli are simple computer generated two-dimensional objects that contain no actual causal event. As such, the causal impression formed by participants can be thought of as a construction of the perceptual system.

Since the groundbreaking research published by Michotte [1] in his seminal book "Perceptual Causality," researchers have strived to understand the boundary conditions under which various perceptual cues available in the environment elicit seemingly "automatic" percepts of causality. Two cues to causality that have received extensive investigation are spatial and temporal contiguity. Specifically, parametric manipulations of spatial gaps and temporal delays between two

*Address correspondence to this author at the Department of Psychology, University of Waterloo, 200 University Avenue West, Waterloo, Ontario, N2L 3G1; Tel: (519) 888-4567 x37197; Fax: (519) 746-8631;

E-mail: jafugels@uwaterloo.ca stimulus movements have been shown to affect the likelihood with which stimulus interactions are rated as causal in a parametric fashion. That is, the larger the temporal delay or the spatial gap that precedes the movement of the second stimulus, the less likely it is that a causal relationship will be reported. These cues have also been found to be critical for other causal impressions, such as the pulling effect (in which a series of objects appears to be pulled by an initial moving object [2]), and the disintegration effect (in which an object disintegrates when a preceding object makes contact with it [3]). Although these effects are quite different, they have in common the finding that people are sensitive to the spatial and temporal cues of the interacting objects, and seem to automatically infer a causal relationship between them if the spatial and temporal cues pass a contiguity threshold. Given these, and host of related findings, several researchers have argued that our visual system is especially tuned to automatically extract causal relations from the environment $[1,4-$ 8]. ${ }^{1}$ That is, the extraction of causality from the visual world appears to occur immediately, involuntarily, and without conscious control.

In the current manuscript, we will first review evidence that the extraction of causal relations from the environment occurs automatically. Following this, we will argue that this automatic extraction of causal relations results in a proliferation of other effects. Specifically, we will review recent findings that have demonstrated how the presence or absence of a perceived causal relationship affects other low-level cognitive processes. Finally, we will propose a new line of research employing event related potential methodologies that shows promise in forging new ground in the quest to under- 
stand the characteristics of perceptual causality and its impact on other cognitive processes.

\section{AUTOMATICALLY EXTRACTING CAUSAL RELATIONS}

As noted above, several researchers have claimed that the perception of causality from simple two-dimensional stimuli seems to arise automatically and is likely an inherent property of the visual system. To evaluate this claim, we must first consider characteristics commonly ascribed to automatic processes. Although theories of automaticity offer differing views on what constitutes an automatic versus a controlled process, common characteristics often ascribed are that automatic processes are stimulus driven [9], fast and effortless [10], unconscious and independent of attention [11]. To what extent does perceptual causality possess these characteristics? Although research has not addressed all of the characteristics ascribed to automaticity, there are numerous sources of evidence that support the claim that the perception of causality can be thought of as an automatic process.

Support for the stimulus driven nature of causal perception was alluded to in the preceding sections of this manuscript. Specifically, numerous experiments, including the original studies conducted by Michotte [1], have demonstrated that the perception of causality appears to be determined by a highly constrained set of visual cues (e.g., spatial and temporal contiguity), and is largely unaffected by topdown processes. Support for this claim comes from studies showing that the perception of causality from launching displays is largely unaffected by experience. For example, Schlottmann and Shanks [12] found that a learned association between a cause (a color change) and an outcome (movement) did not affect people's perception of causality. Rather, their perceptual judgments were based solely on the temporal properties of the collision event.

Second, regarding the time course of causal perception, findings that lend support to the idea that causality can be perceived automatically include those that have shown that the perceptual cues used to generate causal percepts are processed and integrated in an extremely rapid fashion. For example, White [13] argues that one of the most critical perceptual processes that supports causal perception is the temporal integration of dynamic events, which is thought to subserve the detection of change and temporal contiguity. Temporal integration is argued to be one of the key functions of "iconic" processing, which has been shown to occur within a very brief temporal window (i.e., less than $100 \mathrm{~ms}$ ) of stimulus presentation in a variety of domains [14]. Given these findings, it can be argued that the key processes sub-serving perceptual causality unfold within a time frame that precedes conscious awareness.

Finally, support for the unconscious nature of causal perception comes from our own work with patients J.W. and V.P., who have both undergone a corpus callosotomy (i.e., Split-Brain surgery) to help control intractable epilepsy. As visual input to the striate cortex is lateralized to the contralateral hemisphere, these patients provide a unique opportunity to examine the processing abilities of each hemisphere in relative isolation. Previous research with Split-Brain and other patient groups has provided a clear backdrop to test some of the basic phenomenological properties of causal perception. For example, several studies have shown that the right hemisphere possesses a distinct advantage for tasks that require visuospatial [15] and temporal [16] integration. Conversely, the left hemisphere has been shown to have a general advantage for tasks that require linguistic processing [17], generating explanations for events and actions [18], and generating and testing hypotheses about probabilistic relationships [19].

Given these findings, clear hypotheses can be generated regarding potential asymmetries in hemispheric contribution, and the phenomenological characteristics of perceptual causality in the Split-Brain. If perceptual causality can proceed without the aid of left hemispheric processing, this would provide evidence that perceptual causality depends on the rapid integration of visual and temporal cues, in the absence of any more deliberate inferential processing. If, on the other hand, perpetual causality requires the left hemisphere, this would provide evidence that the perception of causality is supported by higher level top-down inferential processing.

In a series of experiments, we [5] found support for the former hypothesis. Specifically, we presented J.W. and V.P. with a series of standard launching events that contained parametric manipulations of spatial and temporal contiguity. We found that the right hemispheres of J.W. and V.P performed similarly to control participants with intact Corpora Callosa; however, the left hemispheres of the same patients performed at chance on this task. That is, the perception of causality was accomplished solely by the right hemisphere of the divided brain. These findings lend support to the hypothesis that causal relations can be extracted from collision events in the absence of basic inferential abilities, thus suggesting a purely perceptual process. ${ }^{2}$ Furthermore, if one accepts the supposition that our conscious experience largely depends on linguistic/inferential mediation [20], which is thought to be the dominion of the left hemisphere [21], these data lend support to the hypothesis that causal perception can proceed in the absence of conscious awareness.

Taken together, these data support the hypothesis that causal relations can be extracted from the environment in a relatively automatic manner akin to other constructive perceptual processes, such as perceptual grouping [22] and illusory contour completion [23]. Given this property of causal perception, it follows that it will be preserved and impact the processing of other basic cognitive processes. In the following section, we will posit that the automatic extraction of causal relations from the environment results in a proliferation of other effects. Specifically, we will review some recent findings that have demonstrated how the presence or absence of a perceived causal relationship affects other lowlevel cognitive processes.

\section{CAUSAL PERCEPTION AND CONTEXT EFFECTS}

Researchers have long known that the perceptual system does not operate in a vacuum. Rather, the context in which a stimulus is presented can significantly influence the processing of that stimulus. This is especially apparent for cases in which the processing of the stimulus under consideration is believed to occur relatively automatically, in that highly automatic processes are thought to be preserved and impact the processing of other stimulus dimensions. A classic example is the Stroop task. Here, word reading is proposed to 
interfere with colour naming due to the relatively greater interference of the more automatic word reading dimension of the task on the less automatic color naming dimension [24]. Similarly, in the Size Congruity paradigm (i.e., the impact that the consistency between the physical size and numerical dimensions of a stimulus has on the time it takes to make a judgment about that stimulus) the physical size of a stimulus interferes with judgments of numerical magnitude [25]. In both of these cases, the more automatic process impacts the processing of other stimulus dimensions.

Numerous examples of the interplay between more automatic perceptual processes and context are also found in auditory and visual perception. For example, in the auditory domain, knowledge of a word has been shown to impact the perception of ambiguous phonemes [26]. Similarly, in the context of music, tonal expectancies have been shown to impact the perception of pitch [27]. In the visual domain, the perceived brightness of an object is determined in part by the perceived luminance [28] and colours [29] of surrounding contextual objects. Similarly, the perceived size of objects is influenced by depth and distance cues [30].

The parallel we would like to draw is that a similar process likely occurs in the perception of causality. Specifically, due to the relatively automatic nature of perceptual causality, this percept will likely be preserved and impact the processing of other stimuli in the context. Given the rapid and relatively automatic nature with which causal impressions seem to be formed, a key question concerns the degree to which such impressions of causality interact with the context in which the stimulus is presented. There are several ways in which context effects in causality may occur. We will focus on two possibilities. First, the presence or absence of a perceived causal relationship may impact the perception of other features of the stimulus in question. Second, the presence or absence of a perceived causal relationship may impact the perception of other stimuli surrounding the causal event.

Consider first the effect of a perceived causal relationship on the perception of other features of the stimulus in question. In a typical Michotte experiment, there are several kinematic features (some observable and some implied) that contribute to the overall causal percept. These include, but are not limited to, motion, speed, direction, and the spatial and temporal contiguities of the interacting objects. Recent studies have shown that individuals' judgments for the kinematic properties of events are affected by their beliefs as to whether a causal relationship does or does not exist between the interacting objects. For example, consider the case of representational momentum. Here, the typical finding is that one's memory for the final position of a moving object is displaced in the direction of the implied motion [31]. That is, if a single object moves from left to right on a screen, followed by a blank screen, participants' memory of the last position of the object is biased towards the right. Several researchers $[32,33]$ have shown that event interactions that imply a causal relationship (e.g., launching displays) result in a significant attenuation of this effect. The explanation for this attenuation is related to participants automatically attributing different properties of force for causal versus noncausal events. Specifically, a "launched" object, in contrast to a "non-launched" single object, is thought to be inert and thus should slow and eventually cease its movement after a collision.

In a more direct test of the impact of the perception of causality on judgments of force, White [34] conducted a series of experiments where participants explicitly judged the force of interacting objects that varied in terms of motion and speed. He found that participants reliably gave higher ratings of force to an object assigned as the causal agent (based on its initial movement) than to an object assigned the role of effect. That is, if a participant believes that an object is the cause, they report it as exerting more force than an object that is believed to be the effect.

Consider now the impact of causal perception of other stimuli in the surrounding context. In a series of experiments Brian Scholl and his colleagues $[35,36]$ provide compelling evidence that the presence of a causal relationship not only influences the perception of other features of the causal stimulus, but also the perception of other stimuli in the context of the causal event. Prior to their studies, the vast majority of research on perceptual causality had focused on how specific properties of the two dynamic objects influenced the perception of causality. Scholl and Nakayama [35, 36], in contrast, found that contextual information can cause an otherwise ambiguous (i.e., non-causal) stimulus, to be judged as causal. To do this, the authors presented participants with either one (no context) or two (context) dynamic visual events. Importantly, for the critical conditions, the test event that was to be judged by participants was identical. Specifically, the test event involved a "launching" dynamic interaction between two objects. However, the key difference was that the second object did not move until the first object passed over and occluded the second object. This "passing event" was presented alone (no context) or synchronously with a "launching stimulus" (context) below the test stimuli. When presented alone without a causal context, participants rated the passing event as causal only $10.7 \%$ of the time. However, when a launching stimulus was presented synchronously with the passing event, the passing event was judged causal $92.1 \%$ of the time. Here, a contiguous collision event (i.e., launching stimulus), when presented alongside an ambiguous stimulus event, made the ambiguous noncausal event appear causal. That is, the causal event appeared to capture the non-causal event and make it appear causal.

In a related study, Scholl and Nakayama [36] found that the presence of a contiguous collision event also produces biased estimates of the temporal and spatial properties of the non-causal event so that they would conform to that of a causal event. They explained this effect in terms of the visual system's tendency to assume non-coincidences in the environment and to thus synchronize events to create a more coherent representation. This explanation is consistent with Purves and Lotto's "empirical theory" of vision [37] where they propose that our perceptual system has evolved to automatically infer stimulus properties based on abstractions and generalizations from past experiences. Here, the visual system's tendency to assume non-coincidences when perceiving causal relations may be the product of our perceptual system automatically constructing a plausible and coherent percept of causality based on the current stimulus features, the context surrounding the event, and generalizations formed from similar stimulus and context interactions expe- 
rienced in the past. Taken together, Scholl and Nakayama $[35,36]$ have provided compelling evidence that the perception of causality doses not proceed in a vacuum. Rather, the perception of causality, and the perception of stimulus relevant features of the causal event, depends on contextual information surrounding the event.

In the present paper, we have reviewed evidence that causal relations are extracted from the environment in a relatively automatic manner. We have argued that it is this automatic property of causal perception that results in the proliferation of context effects wherein the presence or absence of a perceived causal relationship affects the processing of other low-level stimulus features. We now turn our discussion to proposing new lines of research that will potentially further our understanding of the relative automaticity of causality, how the perception of causality is affected by context, and the degree to which the perception of causality can be affected by beliefs, expectations, and new experience.

\section{NEW DIRECTIONS FOR UNCOVERING THE MECHANISMS UNDERLYING THE INTERACTION BETWEEN THE PERCEPTION OF CAUSALITY AND CONTEXT}

Thus far, we have focused our discussion on data employing traditional behavioral measures to assess the degree to which the perception of causality and the proliferation of context effects are the result of the automatic extraction of causal relations from the environment. We have recently been examining the time course with which causal relations are extracted from visual cues using Event Related Potentials (ERPs). ERPs may prove to be an extremely effective methodology to further our understanding of the characteristics of causal processes as they provide a unique window into the time course of the component processes involved in a given task. By providing a continuous measure of stimulus processing, ERPs allow us to determine more directly the stages of processing affected by stimulus manipulations, even in the absence of an overt behavioural response [38]. For example, if a given stimulus manipulation affects early cortical ERP components (e.g., P1 and N1), one can infer that the manipulation affects early sensory and perceptual-level processing. If, however, a stimulus manipulation affects later cortical ERP components (e.g., P3 and N4), one can infer that the manipulation likely affects later post-perceptual (e.g., conceptual) processing [39]. By more directly tapping into the timecourse of this function, one can also gather more evidence regarding the perceptual and inferential components in various causal tasks.

Surprisingly, very little research to date has applied this powerful methodology to the study of causality. In a recent experiment, we [40] took a first step in this direction. Specifically, we examined the degree to which physically plausible versus physically implausible events modulated early versus late ERP components. Participants viewed plausible collision events in which an impacted ball moved horizontally across the screen in a manner consistent with basic laws of physics, and implausible collision events, in which the impacted ball moved upward at an angle of 45 degrees. We found that physically-implausible events elicited a P3 of greater amplitude than physically-plausible events. As the P3 waveform is thought to reflect processes involved in the up- dating of a representation in memory of the stimulus context [41], we took these data to support the claim that violations of expected object interactions, represented schematically [42], influence basic cognitive processes involved in perceiving and monitoring ongoing changes to the environment.

These findings, however, do not allow us to make direct inferences regarding the time course of causal perception per se, as no "non-causal" stimuli were included in the preceding experiment. That is, in both the physically-plausible and physically-implausible displays, the stimuli were both spatially and temporally contiguous and thus did not violate key cues to causality. We are currently following up this work by examining the time course with which spatial and temporal cues are extracted from visual stimuli. This will provide new evidence regarding the relative degree of automaticity of the perception of causality. Specifically, if the perception of causality is indeed an inherent property of the visual system, as we have previously posited (akin to other perceptual phenomena such as perceptual grouping), then manipulations that influence the perception of causality should modulate early cortical components (see Han, Jiang, Mao, Humphreys, \& Qin [22] for a demonstration of the modulation of P1 as a function of perceptual grouping). Once the time course of the extraction of causal relations from spatial and temporal cues is clearly mapped out, we can then begin to examine the attentional characteristics of context effects associated with causal perception. A key question to be addressed will be the degree to which the context effects that arise from the perception of causality penetrate lower levels of visual attention and perception. For example, is the visual systems tendency to assume non-coincidences in the environment (i.e., by linking the context with the central stimulus) a "perceptual" or "conceptual" process? Can one's expectations or beliefs about the context surrounding a causal event impact the perception of that event at a low level (i.e., can the percept of causality be penetrated)? Another key question for future research is to determine the degree to which the mechanisms sub-serving low-level causal perceptual tasks are different than those sub-serving high-level causal reasoning tasks (e.g., inferring causality from covariation-based data). As noted by a reviewer of this manuscript, research on perceptual causality suggests that it might be the lack of perceptual cues in studies of causal induction that lead to the divergent findings in the literature. That is, in the absence of perceptual cues, people must rely on more cognitively taxing induction processes. By including tasks that include causally relevant perceptual features and statistical regularities, future research can provide critical insights into how processing these two dimensions jointly inform causal understanding.

\section{SUMMARY}

Researchers have long been fascinated with the perceptual system's ability to construct high-level percepts from simple stimuli. In recent years, the perception of causality has captured the attention of researchers across multiple disciplines of psychology, including researchers who study basic visual perception, high-level reasoning, and cognitive development. In the current paper, we have reviewed evidence supporting the hypothesis that the perception of causality is an automatic construction of the visual system, and that this property of causality results in the proliferation of a 
wide range of context effects. We have necessarily limited our focus here to low-level cognitive processes. However, many research opportunities exist for higher-level causation as well (see Fernbach, Linson-Gentry, \& Sloman [43] for a recent demonstration of how the belief in a causal relationship impacts temporal processing in a high-level reasoning task). Such research directions will continue to enrich our understanding of the mechanisms that underlie causal knowledge, and how such knowledge interacts with other cognitive processes to enable us to perceive and act upon our environment.

\section{LIST OF ENDNOTES}

${ }^{1}$ Often the notion of automaticity, especially pertaining to the study of perceptual causality, is associated with evidence for or against a modular versus a distributed view of causality [6] and/or whether the processes are innate or learned [44]. In the current paper, we wish to make no theoretical claims or assumptions about what the relative automaticity of causal perception implies about the origins or the representations of the processes associated with causality. The interested reader is referred to Scholl and Tremoulet [6] and Schlottmann [45] for coverage of both sides of this debate.

${ }^{2}$ In the current paper, we provide arguments that the perception of causality is a relatively automatic and lowlevel (i.e., data-driven) visual process. There has been considerable debate in the literature regarding the relative contribution of higher order reasoning processes in causal tasks [46-48]. To date, these debates have primarily focused on causal tasks that are acquired through associative learning and/or contingency-based processes. However, it is entirely possible that top-down influences may contribute and modulate perceptual launching tasks like those discussed in the current paper. This is an important avenue for future research. Indeed, recent research in other areas of cognitive psychology (e.g., Reading, Semantic Priming, etc.) has seriously challenged the view that tasks previously presumed to be automatic operate in the absence of top-down strategic influences [49-51].

\section{ACKNOWLEDGMENTS}

We would like to thank José Perales, Stephanie Solcz, and two anonymous reviewers for comments and helpful suggestions on a previous version of this manuscript. This research was supported by an NSERC discovery grant awarded to Jonathan Fugelsang.

\section{REFERENCES}

[1] Michotte A. The perception of causality. New York: Basic Books 1963.

[2] White PA, Milne A. Phenomenal causality: impressions of pulling in the visual perception of objects in motion. Am J Psychol 1997; 110: 573-602.

[3] White PA, Milne A. Impressions of enforced disintegration and bursting in the visual perception of collision events. J Exp Psychol Gen 1999; 128: 499-516.

[4] Fugelsang J, Roser M, Corballis P, Gazzaniga M, Dunbar K. Brain mechanisms underlying perceptual causality. Cognitive Brain Res 2005; 24: 41-7.

[5] Roser M, Fugelsang J, Dunbar K, Corballis P, Gazzaniga M. Dissociating processes supporting causal perception and causal inference in the brain. Neuropsychology 2005; 19: 591-602.
[6] Scholl BJ, Tremoulet PD. Perceptual causality and animacy. Trends Cogn Sci 2000; 4: 299-309.

[7] Saxe R, Carey S. The perception of causality in infancy. Acta Psychol 2006; 123: 144-65.

[8] White PA. The causal asymmetry. Psychol Rev 2006a; 113: 13247.

[9] Posner MI, Snyder, CRR. Attention and cognitive control. In: Solso RS, Ed. Information processing and cognition: The Loyola Symposium Hillsdale, NJ: Erlbaum 1975; pp. 55-85.

[10] Logan GD. Toward an instance theory of automatization. Psychol Rev 1988; 95: 492-527.

[11] Schneider W, Shiffrin RM. Controlled and automatic human information processing. I. Detection, search and attention. Psychol Rev 1977; 84: 1-66.

[12] Schlottmann A, Shanks DR. Evidence for a distinction between judged and perceived causality. Q J Exp Psychol-A 1992; 44(A): 321-42.

[13] White PA. Causal processing: origins and development. Psychol Bull 1988; 104: 36-52.

[14] Di Lollo V. Temporal integration in visual memory. J Exp Psychol Gen 1980; 109: 75-97.

[15] Corballis PM, Funnell MG, Gazzaniga MS. Hemispheric asymmetries for simple visual judgments in the split brain. Neuropsychologia 2002; 40: 401-10.

[16] Handy TC, Gazzaniga MS, Ivry RB. Cortical and subcortical contributions to the representation of temporal information. Neuropsychologia 2003; 41: 1461-73.

[17] Milner B. Laterality effects in audition. In: Mountcastle VB, Ed Interhemispheric relations and cerebral dominance. Baltimore, MD: Johns Hopkins Press 1962; pp. 177-98.

[18] Gazzaniga MS. Cerebral specialization and interhemispheric communication: Does the corpus callosum enable the human condition? Brain 2000; 123: 1293-1326.

[19] Wolford G, Miller MB, Gazzaniga MS. The left hemisphere's role in hypothesis formation. J Neurosci 2000; 20: 1-4.

[20] Dennett DC. Consciousness explained. Boston, MA: Little, Brown 1991.

[21] Gazzaniga MS. The split-brain revisited. Sci Am 1998; 279: 515.

[22] Han S, Jiang Y, Mao L, Humphreys GW, Qin J. Attentional modulation of perceptual grouping in human visual cortex: ERP studies. Hum Brain Mapp 2005; 26: 199-209.

[23] Corballis PM, Fendrich R, Shapley R, Gazzaniga MS. Illusory contours and amodal completion: Evidence for a functional dissociation in callosotomy patients. J Cogn Neurosci 1999; 11: 459-66.

[24] MacLeod CM. Half a century of research on the Stroop effect: An integrative review. Psychol Bull 1991; 109: 163-203.

[25] Besner D, Coltheart M. Ideographic and alphabetic processing in skilled reading of English. Neuropsychologia 1979; 17: 467-72.

[26] Ganong WF. Phonetic categorization in auditory word perception. J Exp Psychol Human 1980; 6: 110-25.

[27] Marmel F, Tillmann B, Dowling WJ. Tonal expectations influence pitch perception. Percept Psychophys 2008; 70: 853-77.

[28] Williams SM, McCoy AN, Purves D. The influence of depicted illumination on perceived brightness. Proc Natl Acad Sci USA 1998; 95: 13296-13300.

[29] Lotto RB, Purves D. The effects of color on brightness. Nat Neurosci 1999; 2: 1010-14.

[30] Shepard R. Mind sights: Original visual illusions, ambiguities, and other anomalies. San Francisco, CA: WH Freeman 1990.

[31] Freyd JJ, Finke RA. Representational momentum. J Exp Psychol Learn 1984; 10: 126-32.

[32] Hubbard TL, Blessum JA, Ruppel SE. Representational momentum and Michotte's 'launching effect' paradigm. J Exp Psychol Learn 2001; 27: 294-301.

[33] Choi H, Scholl BJ. Measuring causal perception: Connections to representational momentum? Acta Psychol 2006; 123: 91-111.

[34] White PA. Impressions of force in visual perception of collision events: A test of the causal asymmetry hypothesis. Psychon B Rev 2007; 14: 647-52.

[38] Luck SJ. An introduction to the event-related potential technique. Cambridge, MA: MIT Press 2005.

[39] Corbett JE, Enns JT, Handy TC. Electrophysiological evidence for a post-perceptual influence of global visual context on perceived orientation. Brain Res 2009; 1292: 82-92. 
[40] Roser M, Fugelsang J, Handy T, Dunbar K. Gazzaniga M. Representations of physical plausibility revealed by event-related potentials. NeuroReport 2009; 20: 1081-6.

[41] Polich J. Overview of P3a and P3b. In: Polich J, Ed. Detection of change: Event-related potential and fMRI findings. Boston, MA: Kluwer Academic Press 2003; pp. 83-98.

[42] White PA. The role of activity in visual impressions of causality. Acta Psychol 2006b; 123: 166-85.

[43] Fernbach PM, Linson-Gentry P, Sloman SA. Causal beliefs influence the perception of temporal order. In: McNamara DS, Trafton JG, Eds. Proceedings of the 29th Annual Cognitive Science Society. Austin, TX: Cognitive Science Society 2007; pp, 269-74.

[44] Leslie AM, Keeble S. Do six-month old infants perceive causality? Cognition 1987; 25: 265-88

[45] Schlottmann A. Is perception of causality modular? Trends Cogn Sci 2000; 4: 441-42.
[46] De Houwer J, Beckers T, Vandorpe S. Evidence for the role of higher-order reasoning processes in cue competition and other learning phenomena. Learn Behav 2005; 33: 239-49.

[47] Mitchell CJ, De Houwer J, Lovibond PF. The propositional nature of human associative learning. Behav Brain Sci 2009; 32: 183-98.

[48] Waldmann MR, Walker JM. Competence and performance in causal learning. Learn Behav 2005; 33: 211-29.

[49] Stolz JA, Besner D. On the myth of automatic semantic activation in reading. Curr Dir Psychol Sci 1999; 8: 61-5.

[50] Risko EF, Stolz JA, Besner D. Basic processes in reading: Is visual word recognition obligatory? Psychon B Rev 2005; 12: 11924.

[51] Reynolds M, Besner D. Reading aloud is not automatic: Processing capacity is required to generate a phonological code from print. $\mathrm{J}$ Exp Psychol Human 2006; 32: 1303-23.

(C) Fugelsang and Roser; Licensee Bentham Open.

This is an open access article licensed under the terms of the Creative Commons Attribution Non-Commercial License (http://creativecommons.org/licenses/by-nc/3.0/) which permits unrestricted, non-commercial use, distribution and reproduction in any medium, provided the work is properly cited. 